\title{
TÉCNICAS DE ANÁLISE DE MULTICRITÉRIO APLICADAS À MODELAGEM DO ESCOAMENTO SUPERFICIAL NA BACIA DO RIO MUNDAÚ, CEARÁ, BRASIL ${ }^{1}$
}

\author{
MULTI-CRITERIA ANALYSIS TECHNIQUES APPLIED TO SURFACE \\ RUNOFF MODELING IN THE MUNDAÚ RIVER BASIN, CEARÁ, BRAZIL
} TÉCNICAS DE ANÁLISIS MULTICRITERIO APLICADAS AL MODELADO DEL ESCURRIMIENTO SUPERFICIAL EN LA CUENCA DEL RÍO MUNDAÚ, CEARÁ, BRASIL

Augusto César Praciano Sampaio - Instituto Nacional de Pesquisas

Espaciais - São José dos Campos - São Paulo - Brasil

cesarpraciano@hotmail.com

Frederico de Holanda Bastos - Universidade Estadual do Ceará - Fortaleza - Ceará - Brasil
fred.holanda@uece.br

Abner Monteiro Nunes Cordeiro - Universidade Estadual do Ceará - Fortaleza - Ceará - Brasil abnermncordeiro@gmail.com

\section{Resumo}

A seleção e a priorização de áreas que satisfazem um objetivo específico contribuem para otimizar a tomada de decisões estratégicas no planejamento ambiental, a exemplo da identificação de áreas preferenciais para o controle e a redução das taxas de escoamento, apontada como medida eficaz na preservação das bacias hidrográficas. Dessa forma, objetivou-se elaborar um modelo determinístico do potencial de geração de escoamento superficial na bacia do rio Mundaú, Ceará, Brasil. Foram aplicadas técnicas de Análise de Multicritério, dentre as quais: o método do Processo Hierárquico Analítico e da Média Ponderada Ordenada associada à lógica fuzzy. As variáveis uso e cobertura do solo, declividade, grupo hidrológico do solo, precipitação e fluxo acumulado foram selecionadas como critérios de análise, com base no Coeficiente de Escoamento Superficial e no modelo do Soil Conservation System. Os resultados demonstraram bastante flexibilidade do modelo no espaço estratégico de decisão, permitindo identificar as áreas adequadas à geração de fluxos superficiais. Essas áreas estão localizadas na porção oeste da bacia sobre as suas vertentes mais íngremes.

Palavras-chave: Bacia hidrográfica semiárida, Coeficiente de Escoamento Superficial, lógica fuzzy.

\section{Abstract}

The selection and prioritization of areas that conform a specific objective contributes to optimize strategic decision making in environmental planning, such as the identification of preferential areas for the control and reduction of runoff rates, indicated as an effective solution in the preservation of watershed. In this sense, the objective was to make a deterministic model of the potential of surface runoff generation in the Mundaú river basin, Ceará, Brazil. Multi-Criteria Analysis techniques were applied, among them: the Analytic Hierarchy Process and the Ordered Weighted Average associated with fuzzy logic. The variables soil use, land cover, slope, soil hydrological group, precipitation and flow accumulation were selected as criteria of the analysis, 
based on the Runoff Coefficient and the Soil Conservation System model. The results showed a lot of flexibility of the model in the strategic space of decision, being possible to identify the areas most appropriate to the generation of superficial flows. These areas are located in the western of the basin on its steepest slopes. Key words: Semi-arid watershed, Runoff Coefficient, fuzzy logic.

\section{Resumen}

La selección y priorización de áreas que satisfacen una meta específica contribuye a optimizar la toma de decisiones estratégicas en la planificación ambiental, a ejemplo de la identificación de áreas preferenciales para el control y la reducción de las tasas de flujo superficial, indicada como medida eficaz en la preservación de las cuencas hidrográficas. De esa forma, se ha objetivado hacer un modelo determinista del potencial de generación de escurrimiento superficial en la cuenca del río Mundaú, Ceará, Brasil. Se aplicaron técnicas de Análisis Multicriterio, entre las cuales: el método del Proceso de Jerarquía Analítica y la Media Ponderada Ordenada asociada a la lógica fuzzy. Las variables uso y cobertura del suelo, grupo hidrológico del suelo, precipitación pluviométrica y acumulación de flujo se seleccionaron como criterios del análisis, con base en el Coeficiente de Escurrimiento y en el modelo del Soil Conservation System. Los resultados demostraron muy flexibilidad del modelo en el espacio estratégico de decisión, permitiendo identificar las áreas más adecuadas a la generación de flujos superficiales. De manera general, estas áreas se localizan en la porción oeste de la cuenca, sobre sus vertientes más empinadas.

Palabras clave: Cuenca hidrográfica semiárida, Coeficiente de Escurrimiento, lógica fuzzy

Introdução

O advento das técnicas de sensoriamento remoto e geoprocessamento revolucionou a representação cartográfica de fenômenos espaciais, facilitando não só o mapeamento como também o cruzamento de planos de informações georreferenciadas (Câmara; Medeiros, 1998, p. 3). Segundo Florenzano (2008, p. 121), essas tecnologias impulsionaram as metodologias de mapeamento integrado e de síntese. A inclusão da lógica fuzzy (Zadeh, 1965, p. 338) foi outro avanço no mapeamento de fenômenos geográficos.

Entretanto, a construção de modelos matemáticos para simular processos em bacias hidrográficas continua a ser um grande desafio (Miranda, 2010, p. 116). Diante da escassez de dados pluviométricos e fluviométricos, Sartori, Lombardi Neto e Genovez (2005, p. 5) apontam o uso das propriedades fisiográficas da bacia na construção de modelos preditivos do escoamento superficial como uma alternativa viável.

Além das propriedades das chuvas, a ocorrência e a intensidade desse fenômeno estão diretamente ligadas aos aspectos fisiográficos da área de captação, dos quais se destacam a cobertura vegetal, o uso dos solos, a declividade, os tipos de solos, etc. (Casseti, 2005, p. 1; Sartori; Lombardi Neto; Genovez, 2005, p. 5). 
Tendo em vista que o escoamento superficial é um dos principais agentes da erosão laminar e linear, Botelho e Silva (2007, p. 168) ratificam que o controle e a redução das taxas de escoamento têm sido as medidas mais promissoras encontradas para conter problemas relacionados à indisponibilidade hídrica e à erosão dos solos.

Portanto, a determinação de áreas prioritárias para a conservação ambiental, levando em consideração a sua predisposição ao escoamento superficial, é de fundamental importância para a manutenção do equilíbrio dos sistemas fluviais. Segundo Coelho Netto (2003, p. 133), podem-se diferenciar áreas com potencial para escoamentos rápidos em superfície de outras com potencial para infiltração, a partir de sua vocação hidrológica.

Diante do exposto, o presente estudo teve como objetivo avaliar a distribuição espacial do potencial de geração de escoamento superficial na bacia do rio Mundaú, a partir da interação de suas componentes fisiográficas, visando contribuir para a identificação e a priorização de áreas para a aplicação de medidas conservacionistas.

A bacia hidrográfica do rio Mundaú, cujas nascentes encontram-se no maciço de Uruburetama, está localizada no noroeste do estado do Ceará, abrangendo os municípios de Uruburetama, Itapipoca, Tururu e Trairi (Figura 1). Configura-se como uma área de grande complexidade edafoclimática, tendo em vista que seu alto curso se apresenta como um ambiente úmido de exceção no contexto semiárido regional onde está inserida. Os limites da bacia foram definidos a partir do segmento do alto e médio curso do rio Mundaú, correspondente à área de contribuição de aproximadamente $500 \mathrm{Km}^{2}$ do açude Gameleira em Itapipoca - CE. O baixo curso não foi incluído em virtude da inadequabilidade metodológica para regiões estuarinas, sendo necessária uma entrada de dados de maior resolução espacial. 


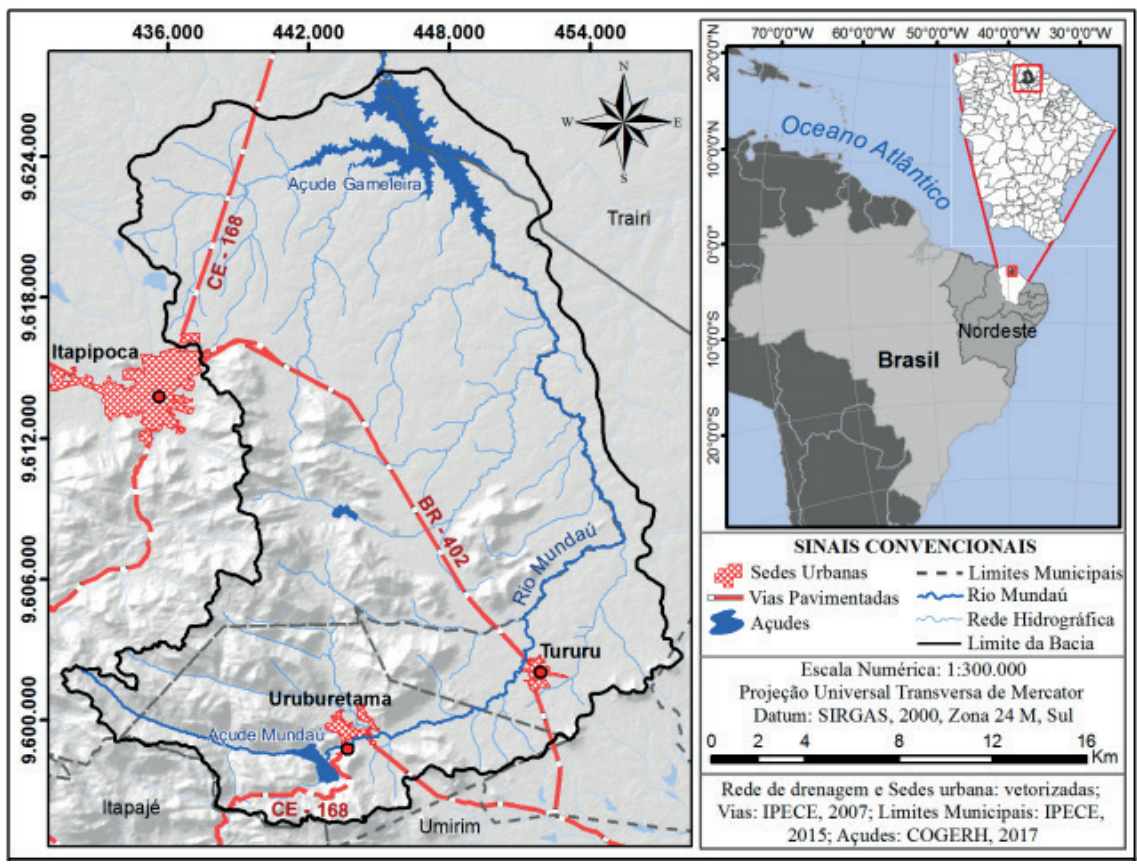

Figura 1 - Mapa de localização da bacia do rio Mundaú

Fonte: Elaborado pelos autores (2018)

\section{Metodologia}

Segundo Malczewski (2004, p. 33), a Análise de Multicritério (AMC) é uma ferramenta capaz de transformar dados espaciais e não espaciais em um produto síntese de saída, por intermédio de regras de decisão, que condicionam a forma como os dados de entrada vão se relacionar para atender ao objetivo da análise. A AMC diminui a subjetividade na tomada de decisão, por meio de processos cognitivos mais robustos (Fitz, 2008, p.142).

Portanto, a AMC foi aplicada na ponderação dos fatores declividade, uso e cobertura do solo, grupo hidrológico do solo, precipitação e fluxo acumulado, na definição das áreas mais aptas ao escoamento superficial. Dentre os métodos que envolvem a AMC, foram aplicados: o Analytic Hierarchy Process (AHP), na definição dos pesos de importância entre os 
critérios; a lógica fuzzy, na realocação dos valores dos critérios, e a Média Ponderada Ordenada (MPO), na agregação dos critérios em um único mapa síntese. O Quadro 1 esquematiza a sequência de métodos empregados na construção do modelo.

Moreira et al. (2001, p. 2) classificam os modelos de aplicação da AMC quanto ao tratamento dos dados de entrada em: modelos teóricos, quando se fundamentam em conceitos estabelecidos da física e da matemática, e modelos empíricos, cuja construção tem base em relações heurísticas ou em estatísticas definidas pelos especialistas responsáveis.

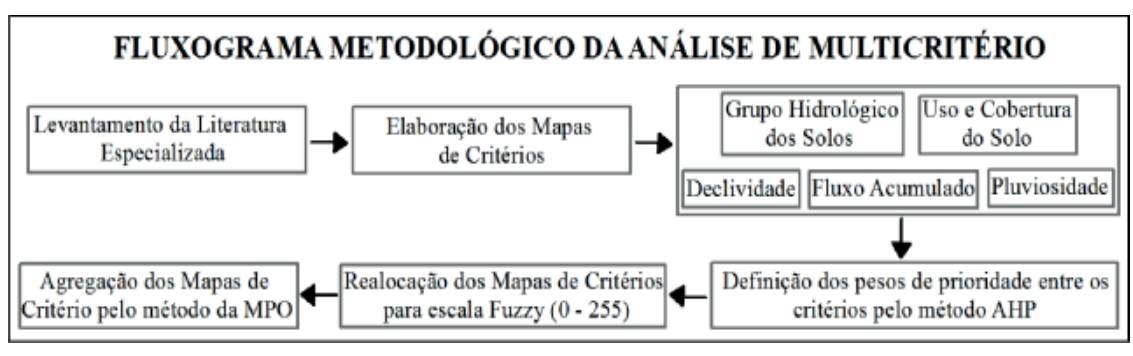

Quadro 1 - Esquema metodológico

Fonte: Elaborado pelos autores (2018)

O AHP, proposto por Saaty (1977, p. 234), é um método usado para ponderar os fatores envolvidos na tomada de decisão, atribuindo pesos de importância entre os critérios, mediante uma matriz de comparação pareada. A matriz é elaborada pela comparação de cada par de critérios, definindo o grau de importância (peso de prioridade) do critério da linha em relação ao da coluna, para o objetivo da análise. Assim, o peso de prioridade varia de 1, para igual importância entre os pares, a 9, para preferência máxima ao critério da linha.

A descentralização do problema de decisão, a organização dos critérios de acordo com sua importância para o objetivo da análise e o teste da consistência dos julgamentos são algumas das vantagens do método AHP (Saaty, 1990, p. 12; Moreira et al., 2001, p. 28).

Após a definição dos pesos de prioridades entre os critérios, a matriz de comparação $(A)$ é normalizada, dividindo-se cada um de seus elementos pelo total de sua respectiva coluna. A matriz normalizada resultante é usada para o cálculo do vetor de prioridades relativas $(w)$, que consiste na média aritmética de cada linha da matriz. 
Para Saaty (1990, p. 14), a consistência dos julgamentos de prioridades é verificada pela Razão de Consistência (RC), que deve ser inferior a $0,1(10 \%)$ para que sejam considerados coerentes. O valor de RC é calculado pela razão entre o Índice de Consistência (IC) e o Índice Randômico (IR). Este último é tabelado para uma série de matrizes quadradas de ordem “n”, conferida em Saaty (1987, p. 171). O IC pode ser calculado pela Equação 1, onde: $\lambda \max$ é o maior autovalor da matriz $A$ e $n$ é a ordem da matriz $A$.

$$
I C=\frac{\lambda_{\max }-n}{\mathrm{n}-1} I C=\frac{\lambda_{\max }-n}{\mathrm{n}-1}
$$

Portanto, é necessário obter primeiro o valor de $\lambda \max$, que é calculado pela média aritmética do vetor resultante da Equação 2, onde: $A$ é a matriz de comparação pareada; $w$ é o vetor de prioridades relativa, e $\lambda \max$, o maior autovalor da matriz $A$.

$$
A \cdot w=\lambda_{\max } \cdot w A \cdot w=\lambda_{\max } \cdot w
$$

Uma vez calculados os pesos de prioridades e verificada a consistência dos julgamentos, há a necessidade de estabelecer uma escalapadrão aos atributos de cada critério, quanto à sua adequação ao objetivo da análise. Nesse sentido, a lógica fuzzy, proposta por Zadeh (1965, p. 338), pode ser aplicada. Segundo Burrough e Mcdonnell (1998, p. 267), os conjuntos fuzzy permitem construir modelos para representar a transição gradual da condição de pertinência a não pertinência a um determinado conjunto.

Um conjunto fuzzy (A) em X é definido matematicamente por um par ordenado (Equação 3), onde x é o elemento do universo $\mathrm{X}$ e $\mu \mathrm{A}$ (x) é o grau de pertinência de $\mathrm{x}$ em A. O valor de $\mu \mathrm{A}$ (x) é um número definido por um intervalo na escala fuzzy.

$$
\left.\left.A=\left\{x, \mu_{A}(x)\right)\right\}, x \in X A=\left\{x, \mu_{A}(x)\right)\right\}, x \in X
$$


Essa escala pode ser representada por um intervalo de 0 a 255 (níveis de bit), sendo que o grau de pertinência fuzzy de um elemento é definido por uma função de pertinência, cujas mais comuns são as lineares e as senoidais (Ferreira et al., 2004, p. 9; Burrough; Mcdonnell, 1998, p. 270). A Equação 4 descreve uma função de pertinência linear crescente, enquanto a Equação 5 representa uma função senoidal crescente.

$$
\begin{aligned}
& F p(z)=\left[\frac{\left(z-L_{i}\right)}{L_{s}-L_{i}}\right] \cdot 25 \mathrm{~s} F p(z)=\left[\frac{\left(z-L_{i}\right)}{L_{s}-L_{i}}\right] \cdot 255 \\
& F p(z)=\left(\cos ^{2} \alpha\right) \cdot 255 F p(z)=\left(\cos ^{2} \alpha\right) \cdot 255
\end{aligned}
$$

Em que: $\alpha=\left[1-\left(\frac{z-L_{i}}{L_{s}-L_{i}}\right)\right] \cdot \frac{\pi}{2} \alpha=\left[1-\left(\frac{z-L_{i}}{L_{s}-L_{i}}\right)\right] \cdot \frac{\pi}{2}$

Em que: $\mathrm{Fp}(\mathrm{z})$ é a função de pertinência de um elemento z em um conjunto fuzzy A; z é o valor que se deseja realocar; Li é o limite inferior do intervalo que se deseja realocar; $L s$ é o limite superior desse intervalo, e $\pi$ é o valor de pi, igual a $180^{\circ}$. Assim, $F p(z)=0$, para valores abaixo do $L i$, e Fp(z) $=255$, para valores acima do Ls. Na Figura 2, observa-se o gráfico das funções de pertinência fuzzy linear e senoidal, crescentes.

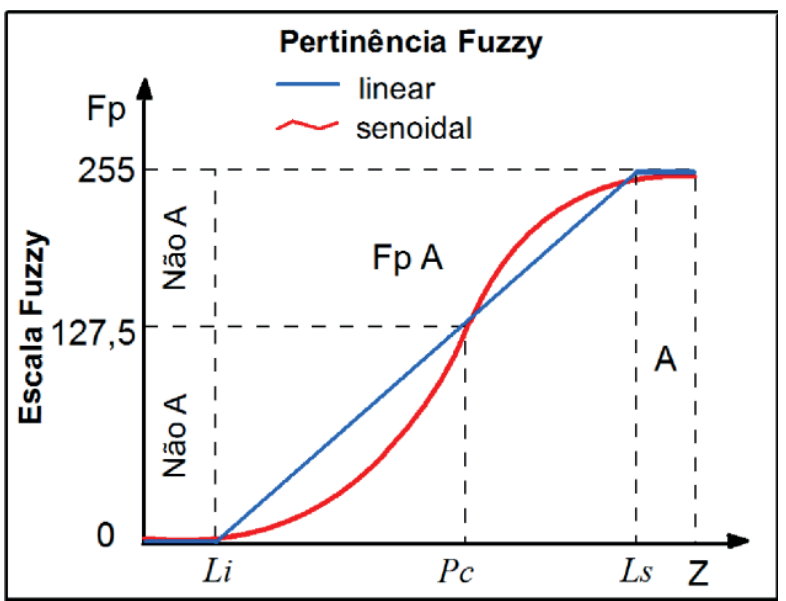

Figura 2 - Gráfico das funções de pertinência fuzzy linear e senoidal (Em que:

$\mathrm{Li}$, é o limite inferior; Ls é o limite superior; Pc é o ponto de crossover) 
A MPO consiste no método de agregação dos critérios e tem como particularidade a atribuição de um segundo grupo de pesos, além dos pesos de prioridades, denominados de pesos de ordenação (Eastman, 2001, p. 11). Nesse sentido, os planos de informações de entrada são combinados usando uma média aritmética ponderada com os pesos de prioridades, conforme a Equação 6, onde: MP é a média ponderada; Wij é o peso dos atributos $i$ pertencente ao plano de informação $j$, na escala fuzzy, e yj é o peso do plano de informação $j$, calculado pelo método AHP.

$$
M P=\frac{\sum_{i=1}^{n} w_{i j} y_{j}}{\sum_{i=1}^{n} y_{j}} M P=\frac{\sum_{i=1}^{n} w_{i j} y_{j}}{\sum_{i=1}^{n} y_{j}}
$$

Em seguida, são aplicados os pesos de ordenação. Para Eastman (2001, p. 11), esses pesos estão relacionados à ordem de compensação que cada um dos critérios assume em cada ponto do mapa. Assim, os pesos de ordenação ajustam o modelo de decisão no espaço entre os extremos de risco mínimo (AND) e máximo (OR).

Portanto, os pesos de ordenação são distribuídos de forma crescente, de modo que o menor escore entre os critérios de determinado ponto do mapa recebe o primeiro peso de ordenação, o segundo menor escore recebe o segundo peso de ordenação, e assim por diante. Dessa forma, o risco é ajustado para o mínimo, quando os primeiros pesos de ordenação (atribuídos aos critérios mais limitantes) recebem os maiores valores, e para o máximo, quando os últimos pesos de ordenação (atribuídos aos critérios de maior potencial) recebem os maiores valores (Eastman, 1998, p. 192, 193).

\section{Procedimentos técnicos e operacionais}

A seleção e a comparação dos critérios estão fundamentadas no modelo hidrológico do Soil Conservation Service (SCS, 1972, p.101) e no modelo determinístico proposto por McCuen (1998, p. 376), denominado de coeficiente de escoamento superficial, que define a porcentagem da precipitação convertida em escoamento superficial, em função do grupo hidrológico do solo, do tipo de uso e da cobertura do solo e declividade.

Nesse sentido, foi aplicado o método AHP com os valores de prioridades sugeridos pelos desvios-padrão das médias dos coeficientes 
de escoamento superficial de cada classe definida por McCuem (1998, p. 376). Esses valores demonstraram a variação dos valores em torno da média para cada critério em particular e, com base na razão entre cada um deles, foi definido o escore de importância relativa entre os critérios. Os fatores pluviosidade e fluxo acumulado foram adicionados aos anteriores e relacionados conforme é indicado pela matriz de comparação exibida no Quadro 2, que também mostra o vetor de prioridade relativa e os parâmetros que medem a consistência dos julgamentos.

\begin{tabular}{|c|c|c|c|c|c|c|}
\hline \multicolumn{6}{|c|}{ MATRIZ DE COMPARAÇÃO PAREADA (A) } & \multirow{2}{*}{$\begin{array}{c}\text { Vetor de } \\
\text { Prioridades } \\
\text { (w) }\end{array}$} \\
\hline & pluviosidade & $\begin{array}{c}\text { uso } \\
\text { cobertura*1 } \\
\end{array}$ & declividade & $\begin{array}{c}\text { Grupo } \\
\text { hidrológic*2 }\end{array}$ & $\begin{array}{c}\text { fluxo } \\
\text { acumulado }\end{array}$ & \\
\hline pluviosidade & 1 & 1 & 2 & 3 & 4 & 0,31 \\
\hline uso e cobertura*1 & 1 & 1 & 2 & 3 & 4 & 0,31 \\
\hline declividade & $1 / 2$ & $1 / 2$ & 1 & 2 & 3 & 0,18 \\
\hline grupo hidrológic*2 & $1 / 3$ & $1 / 3$ & $1 / 2$ & 1 & 4 & 0,13 \\
\hline fluxo acumulado & $1 / 4$ & $1 / 4$ & $1 / 3$ & $1 / 4$ & 1 & 0,06 \\
\hline \multicolumn{7}{|c|}{ CONSISTÊNCIA DOS JULGAMENTOS } \\
\hline \multirow{5}{*}{$\begin{array}{l}\text { Vetor de Pesos } \\
\qquad \text { (A.w) }\end{array}$} & 1,61 & \multirow{5}{*}{$\begin{array}{c}\text { Vetor de } \\
\text { Consistência } \\
(\text { Aw / w) }\end{array}$} & 5,19 & $\lambda \max$ & \multicolumn{2}{|c|}{5,16} \\
\hline & 1,61 & & 5,19 & IC & \multicolumn{2}{|c|}{0,04} \\
\hline & 0,93 & & 5,17 & IR & \multicolumn{2}{|c|}{1,2} \\
\hline & 0,6646 & & 5,11 & $\mathrm{RC}$ & \multicolumn{2}{|c|}{0,03 ou $3 \%$} \\
\hline & 0,3069 & & 5,12 & & & \\
\hline
\end{tabular}

Quadro 2 - Matriz de comparação pareada, vetor de prioridades e consistência dos julgamentos ( ${ }^{* 1}$ uso e cobertura dos solos; ${ }^{* 2}$ grupo hidrológico dos solos)

Fonte: Elaborado pelos autores (2018)

O mapa de uso e cobertura do solo foi confeccionado com a técnica de classificação supervisionada, classificador MAXVER, do tipo pixel a pixel, em que foram coletadas amostras de treinamento do algoritmo para as classes de uso e cobertura do solo adaptadas de McCuem (1998 p. 376), quais sejam: solo florestado (caatinga), campo (solo exposto), áreas urbanizadas e solo cultivado.

Utilizou-se uma imagem do sensor OLI/LDCM (Operational Land Imager/Thermal Infrared Sensor) do satélite Landsat 8, com data de imageamento de 26 de agosto de 2016, correspondendo ao período seco do ano, quando as condições reais de cobertura são mais evidentes. Foi 
utilizada uma composição falsa cor com as bandas 6, 5, 4, fusionadas (Pansharpening) à banda pancromática, com resolução espacial de 15 metros.

Conforme Eastman (2009, p. 98), as variáveis qualitativas não podem ser realocadas usando uma função de pertinência aos conjuntos fuzzy. Dessa forma, foram realocadas, atribuindo-se valores específicos na escala fuzzy, definidos com base no coeficiente de escoamento superficial de cada classe definido por McCuem (1998, p. 376).

O mapa de tipo hidrológico dos solos foi elaborado pela classificação do mapa de solos do Zoneamento Agrícola do Estado do Ceará (Ceará, 1988, p.1), na escala de 1:200.000. A nomenclatura dos solos foi atualizada para o Sistema Brasileiro de Classificação dos Solos (SiBCS) (EMBRAPA, 2013, p. 78).

As classes de solos foram reclassificadas quanto ao seu tipo hidrológico com base na proposta de Sartori (2004, p. 26), para os solos do Brasil, que se baseiam na classificação do SCS (1972, p. 101). As classes dos grupos hidrológicos dos solos também foram realocadas atribuindo-se valores específicos na escala fuzzy, com base no coeficiente de escoamento superficial.

O mapa de declividade foi gerado pelo processamento automático do Modelo Digital de Elevação (MDE) da Shuttle Radar Topography Mission (SRTM), com 30 metros de resolução espacial. O critério declividade foi realocado usando uma função de pertinência fuzzy senoidal crescente, com pontos de inflexão mínimo de $0^{\circ}$ e máximo de $45^{\circ}$. Assim, os valores acima de $45^{\circ}$ receberam o valor de 255 . O mapa de pluviosidade foi elaborado pela interpolação das médias históricas anuais de precipitação de 1986 a 2015 para os postos de Irauçuba, Itapajé, Itapipoca, Miraíma, Pentecoste, São Luís do Curu, Umirim, Uruburetama e Tururu (Ceará, 2017, p.1).

Foi utilizada a krigagem ordinária, que se baseia no semivariograma para estabelecer a influência de cada ponto amostral na estimativa dos pontos desconhecidos. A geração do semivariograma foi associada à aplicação de um modelo de ajuste gaussiano (Figura 3), capaz de expressar melhor a variação espacial do fenômeno estudado.

O resultado foi submetido ao teste de validação cruzada. Segundo Kerry e Oliver (2007, p. 389), o teste consiste em retirar sistematicamente cada ponto amostrado e calcular o resíduo entre o valor real e o valor estimado. Dessa forma, o modelo apresentou Média Quadrática do Erro (MQE) igual a 152. O critério pluviosidade foi realocado por uma função 
de pertinência linear crescente, com ponto mínimo de 829 mm e máximo de $1053 \mathrm{~mm}$.

O fluxo acumulado foi gerado pelo processamento automático do MDE/SRTM, do qual foi gerado o raster de acúmulo do fluxo. Este último foi utilizado para a extração dos canais de fluxo acumulado, definidos por um limiar de $450.000 \mathrm{~m}^{2}$ de área de contribuição. O intervalo de $0 \mathrm{a}$ $450.000 \mathrm{~m}^{2}$ foi ajustado por uma função de pertinência senoidal crescente, com pontos de inflexão em 0 e $9.000 \mathrm{~m}^{2}$.

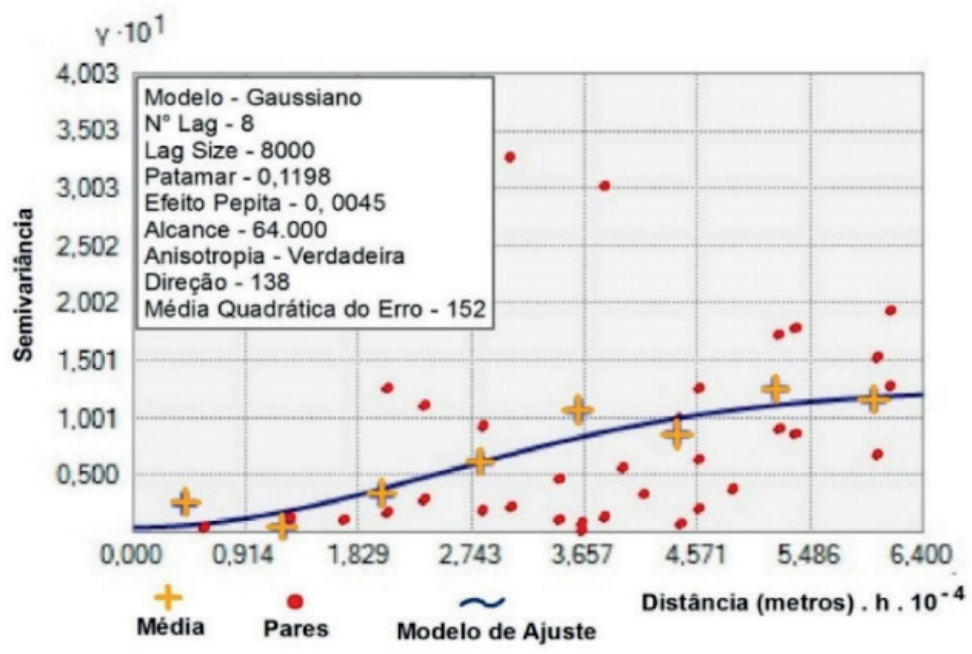

Figura 3 - Semivariograma experimental para aplicação da krigagem

Fonte: Elaborado pelos autores (2018)

Com os mapas de critérios realocados para a escala fuzzy, foram gerados dois mapas com o método da MPO. O primeiro com tendência para o risco mínimo (37,5\%), recebendo os pesos de ordenação 0,$30 ; 0,25$; 0,$20 ; 0,15 ; 0,10$, do critério com escore mais baixo para o mais alto. $\mathrm{O}$ segundo com compensação plena (100 \%) e risco médio, ou seja, os pesos de ordenação foram distribuídos com os mesmos valores, sendo, assim, anulados. 


\section{Resultados e discussões}

A declividade da bacia do rio Mundaú varia entre 0 e 55 graus, sendo que os declives mais acentuados estão localizados nas vertentes do maciço de Uruburetama. Nessa região, o gradiente só é suavizado nas planícies alveolares e nas reentrâncias erosivas. Por outro lado, na superfície erosiva rebaixada, predomina um relevo do tipo plano a suavemente ondulado. Nessa unidade, os maiores declives ficam a cargo dos afloramentos rochosos.

A precipitação aumenta significativamente em direção ao litoral e na porção leste do maciço de Uruburetama. Segundo Brandão et al. (2003, p. 21), em decorrência do aumento da altitude sobre o maciço, ocorre um aumento significativo da pluviometria, em função dos aspectos orográficos e de sua posição em relação aos ventos úmidos do litoral.

O fluxo acumulado apresenta um espaçamento maior para se tornar canalizado no maciço de Uruburetama, em virtude do maior comprimento das vertentes. A área de contribuição para o acúmulo de fluxo canalizado varia de $90 \mathrm{~m}^{2}$ a $450.000 \mathrm{~m}^{2}$. Na Figura 4, pode-se observar a variação da declividade, da pluviosidade e do fluxo acumulado na bacia.

Quanto aos solos, no domínio do maciço de Uruburetama, predominam os Argissolos Vermelho Amarelo (PVA), profundidade média a alta, que também podem ser observados na baixa encosta (Ceará, 1988, p. 1; Brandão el al., 2003, p. 29). Nas encostas mais íngremes, ocorrem os Neossolos Litólicos (RL), associados a afloramentos de rocha. Esses solos são rasos, pouco desenvolvidos e não hidromórficos. 


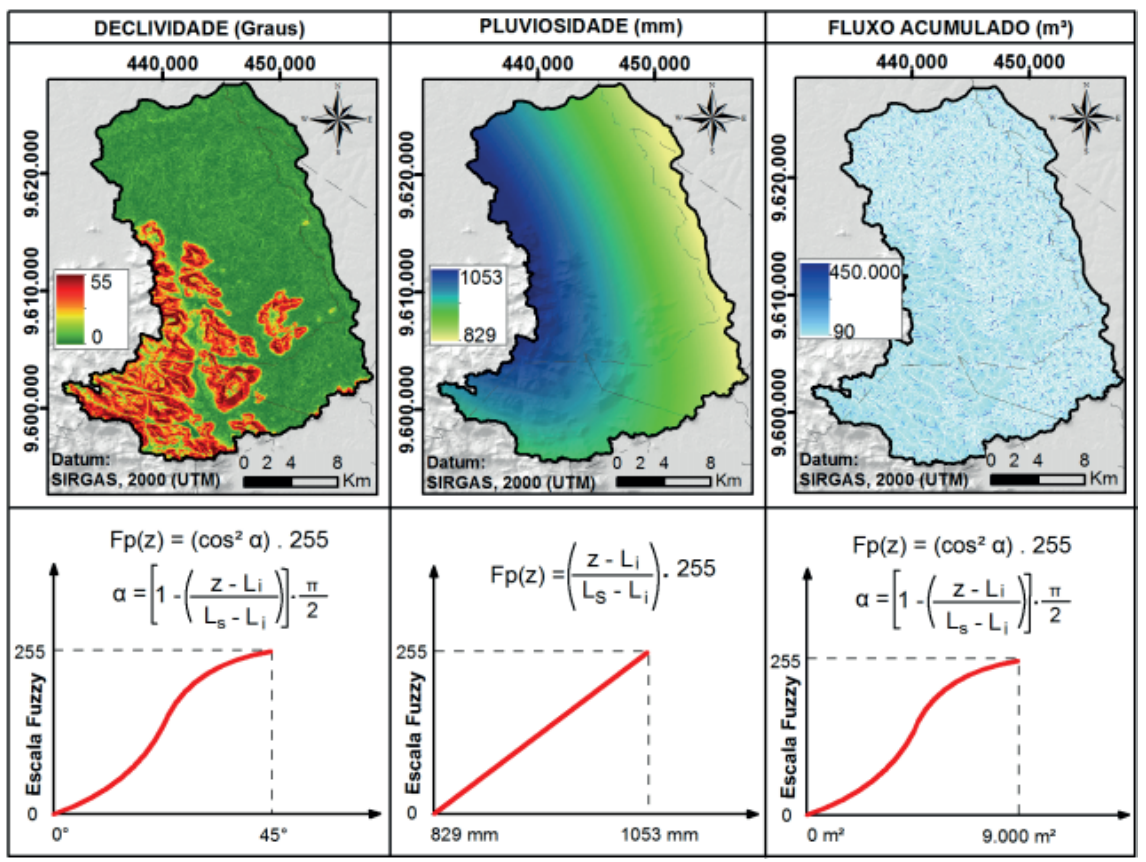

Figura 4 - Mapas dos critérios: declividade, pluviosidade e fluxo acumulado, e suas respectivas funções de pertinência ao conjunto fuzzy

Fonte: Elaborado pelos autores (2018)

Por outro lado, no domínio da superfície erosiva rebaixada, verificam-se os Neossolos Regolíticos (RR) e Planossolos Háplicos e Nátricos (SX + SN), moderadamente rasos (Ceará, 1988, p. 1; Brandão et al., 2003, p. 29). No setor nordeste da bacia, ocorre uma faixa estreita pertencente aos tabuleiros costeiros. Essa unidade é coberta por Latossolos Amarelos (LA) de profundidade alta (Ceará, 1988, p. 1). O Quadro 3 contém a classificação dos solos da bacia quanto ao seu tipo hidrológico e a seu significado para o escoamento superficial. 


\begin{tabular}{|c|c|c|}
\hline Classificação dos Solos SiBCS (2013) & $\begin{array}{c}\text { Grupo Hidrológico } \\
\text { (SCS) }\end{array}$ & $\begin{array}{c}\text { Potencial para o Escoamento } \\
\text { Superficial }\end{array}$ \\
\hline Argissolos Vermelho Amarelo (PVA) & C & Alto \\
\hline Neossolos Litólicos (RL) & D & Muito Alto \\
\hline Neossolos Regolíticos (RR) & D & Moderado \\
\hline Latossolos Amarelos (LA) & B & Muito Alto \\
\hline Planossolos Háplicos e Nátricos (SX + SN) & D & \\
\hline
\end{tabular}

Quadro 3 - Tipo hidrológico dos solos da bacia do rio Mundaú

Fonte: Elaborado pelos autores (2018), fundamentado em Ceará (1988) e Sartori (2004)

Os tipos de uso e cobertura do solo da bacia estão muito associados com os seus aspectos edáficos e climáticos. Na Figura 5, podem ser observados os mapas de uso e cobertura, o grupo hidrológico dos solos da bacia, além da álgebra de mapas aplicada.

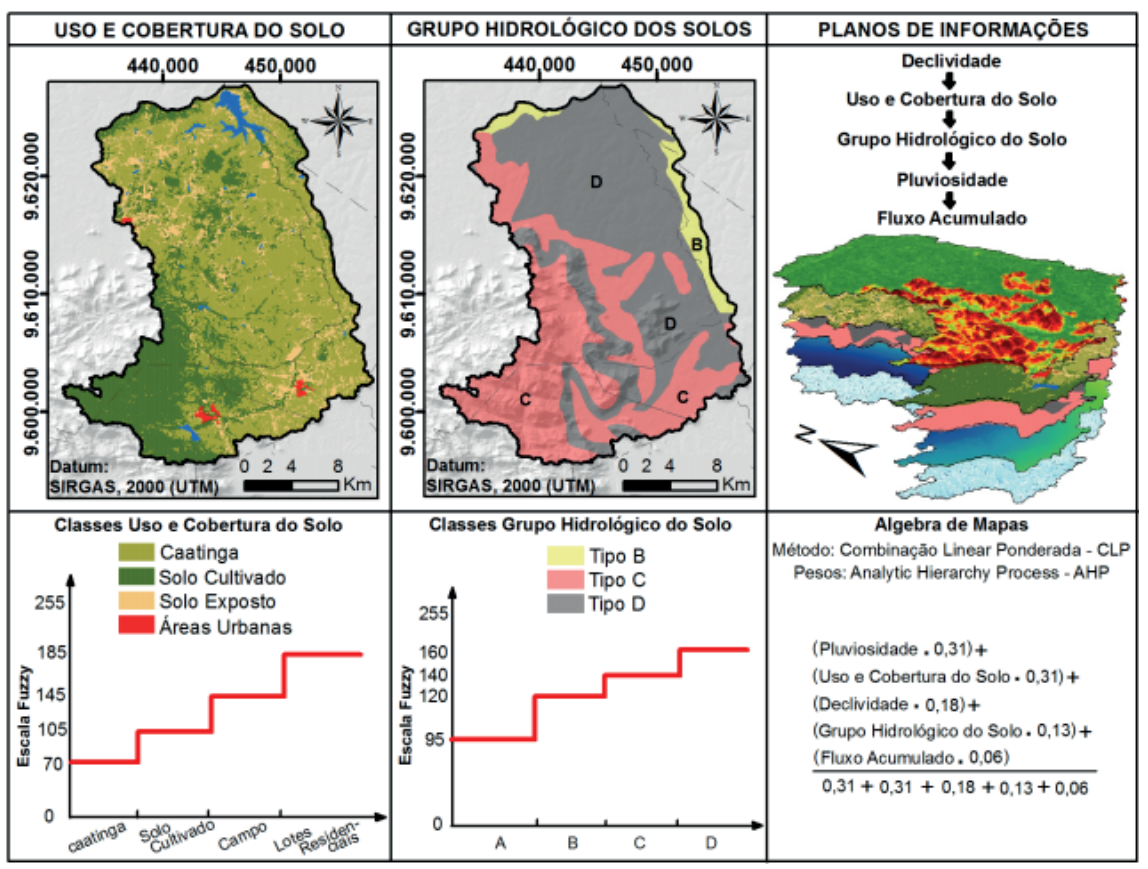

Figura 5 - Mapas dos critérios uso e cobertura do solo e grupo hidrológico dos solos e suas respectivas escalas fuzzy / Álgebra de Mapas 
A atividade da bananicultura substitui, em maior parte, a cobertura vegetal nativa sobre as vertentes orientais do maciço de Uruburetama, tendo em vista o aproveitamento das melhores condições edáficas e de umidade. Quando em encostas íngremes, essa atividade pode provocar a perda de estabilidade das encostas pelo aumento da produção de escoamento superficial, aliado à supressão da cobertura vegetal.

A caatinga arbustiva ocupa a maior parte do espaço não usado para o cultivo, predominando na superfície pediplanada, e também nas cotas mais baixas do maciço. Segundo Souza e Oliveira (2006, p. 90), a floresta subúmida colonizava o maciço, mantida pelo mesoclima de altitude, no entanto, quase não se encontram remanescentes desta.

A agregação dos fatores resultou em um modelo do potencial de geração de escoamento superficial na bacia do rio Mundaú, com valores no intervalo fuzzy de 0 a 255. Os valores foram classificados usando o classificador de quebras naturais Jenks (1977, p.22).

No mapa com a compensação plena e risco médio (Figura 6), observa-se que as vertentes a nordeste do maciço de Uruburetama, porção leste da bacia, e as áreas rebaixadas próximas à cidade de Itapipoca apresentam as maiores aptidões para o escoamento superficial, pela combinação de fatores como declividade e precipitação elevada com os Argissolos e Neossolos Litólicos, onde 18\% da área da bacia apresenta potencial alto/altíssimo e 42,5\%, potencial de escoamento superficial no intervalo moderado. 


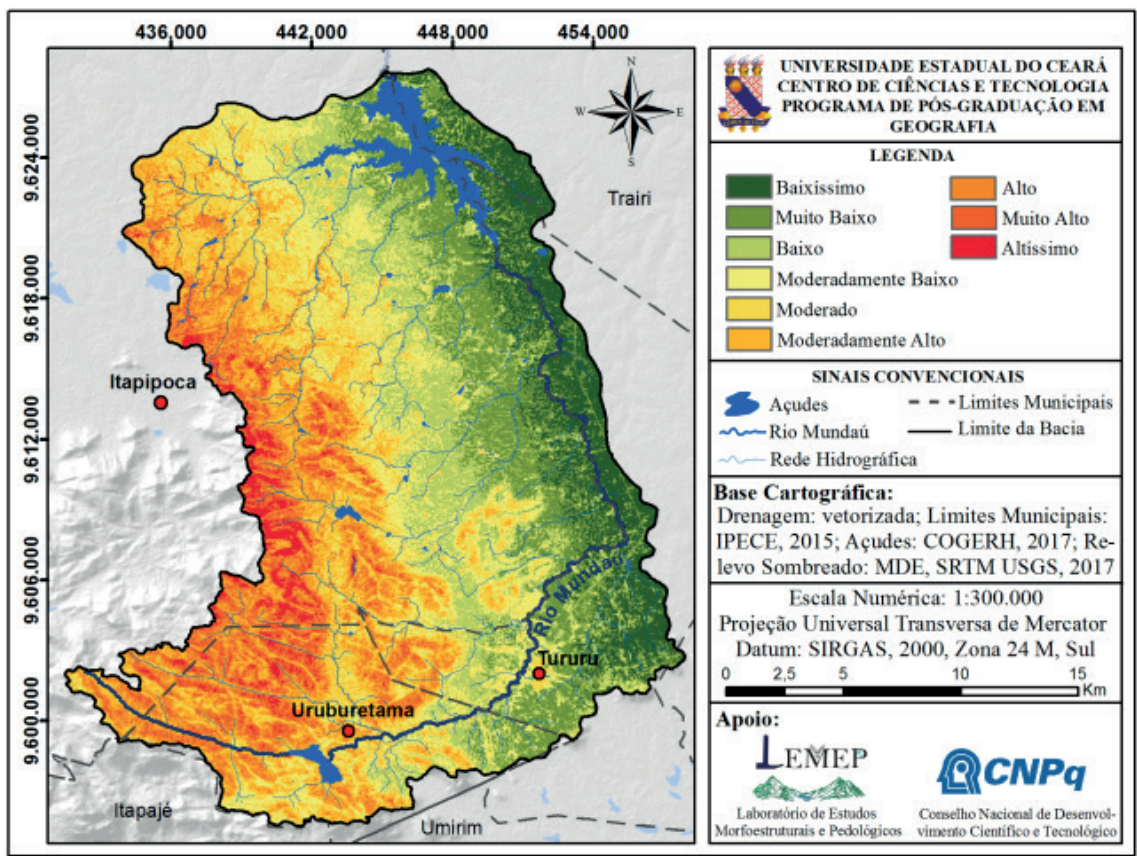

Figura 6 - Mapa do potencial de escoamento superficial na bacia do rio Mundaú pelo método da Média Ponderada Ordenada - risco médio (50\%) e compensação de $100 \%$

Fonte: Elaborado pelos autores (2018)

As regiões mais cultivadas sobre as vertentes úmidas do maciço de Uruburetama apresentaram potencial de moderado a alto na geração de fluxos superficiais. De maneira geral, o potencial de escoamento superficial decresce para o leste da bacia, evidenciando a forte influência da declividade e da pluviosidade, em detrimento das demais variáveis.

O mapa de risco baixo (37,5\%) (Figura 7) mostrou maior correlação com o critério declividade, classificando as vertentes mais íngremes ao leste do maciço de Uruburetama como as áreas de maior potencial para a geração de escoamento superficial. 


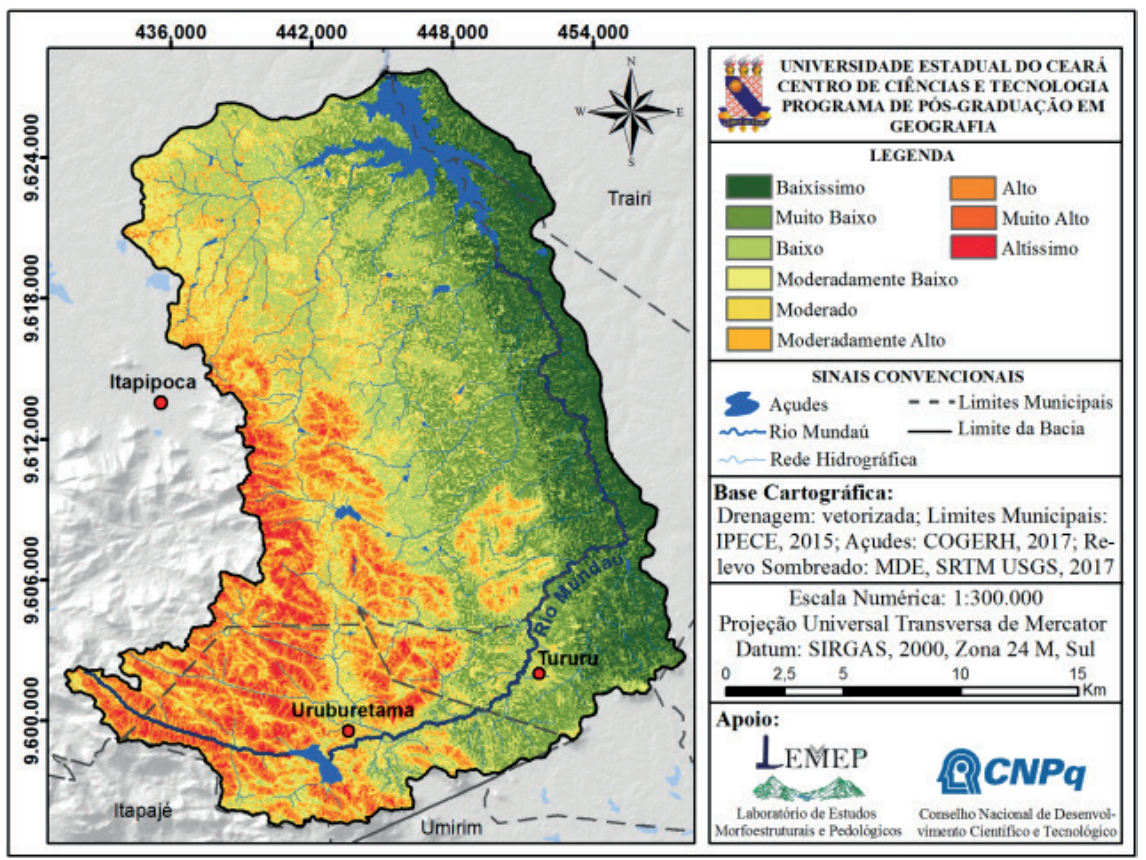

Figura 7 - Mapa do potencial de escoamento superficial na bacia do rio Mundaú pelo método da Média Ponderada Ordenada - risco baixo $(37,5 \%$ e e compensação de $92 \%$

Fonte: Elaborado pelos autores (2018)

Dessa forma, o critério declividade demonstrou ser o mais seguro para definir o potencial de escoamento superficial na área de estudo. Isso se deve em parte ao fato de os maiores declives estarem diretamente relacionados com outros fatores que reafirmam a geração de fluxos superficiais, como a precipitação elevada, a maior concentração de fluxos e a presença de solos rasos, evidenciando um mecanismo de retroalimentação positiva.

\section{Considerações finais}

Os resultados reforçam a importância das técnicas de AMC associadas à lógica fuzzy para o mapeamento integrado com diferentes objetivos, como a priorização de áreas e a análise de susceptibilidade. 
O método AHP permitiu dividir o problema de decisão, diminuindo a influência da subjetividade no modelo final.

O modelo apresenta flexibilidade para ser ajustado com outras regras de decisão, a exemplo do grau de importância relativa entre os critérios de análise. Essas regras podem ser estabelecidas com base em parâmetros definidos por relacionamentos estatísticos ou empíricos que revelem as especificidades de cada região.

A MPO resultou na construção de um modelo flexível, permitindo ajustá-lo quanto ao risco assumido na tomada de decisão e controlar a compensação entre os fatores. Uma vantagem da metodologia é que ela pode ser aplicada em qualquer software de Sistemas de Informações Geográficas (SIG) que permita operações algébricas com dados matriciais.

\section{Nota}

1 Esta pesquisa é subsidiada por bolsa de mestrado concedida pelo Conselho Nacional de Desenvolvimento Científico e Tecnológico (CNPq), com data de vigência entre 1ㅇ de março de 2016 a 28 de fevereiro de 2018.

\section{Referências}

BOTELHO, R.G. M.; SILVA, A. S. da. Bacia hidrográfica e qualidade ambiental. In: VITTE, A. C.; GUERRA, A. J. T. (Orgs). Reflexões sobre a geografia física no Brasil. 2 ed. Rio de Janeiro: Bertrand Brasil, 2007. p. 153-192.

BRANDÃO, R. L.; COLARES, J. Q. dos S.; GOMES, F. E. M.; SOUZA, M. J. N. Zoneamento geoambiental da região de Irauçuba. Fortaleza: CPRM, 2003.

BURROUGH, P. A.; MCDONNELL, R. A. Principles of geographical information systems. Oxford: Oxford University Press, 1998.

CÂMARA, G.; MEDEIROS, J. S. Princípios básicos em geoprocessamento. In: ASSAD, E. D.; SANO, E. E. (Eds.) Sistema de Informações Geográficas: aplicações na agricultura. 2 ed., Brasília: EMBRAPA - SPI / CPAC, 1998. p. 3-11.

CASSETI, V. Geomorfologia. [S.l.], 2005. Disponível em: <http://www.funape. org.br/geomorfologia/>. Acesso em: 11 nov. 2017.

CEARÁ. Fundação Cearense de Meteorologia e Recursos Hídricos (FUCEME). Fortaleza: FUNCEME, 2017. Séries históricas. Disponível em: < http://www. funceme.br/index.php/areas/23-monitoramento/meteorológico/572-postospluviométricos\#site >. Acesso em: 25 set. 2017.

. Secretaria de Agricultura e Reforma Agrária - SEARA / Instituto do Desenvolvimento Agrário do Ceará Zoneamento Agrícola do Estado do CearáIDACE. Zoneamento Agrícola do Estado do Ceará. Fortaleza, 1988. 
COELHO NETO, A. L. Hidrologia de encosta na interface com a geomorfologia. In: GUERRA A. J. T.; CUNHA, S. B. (Orgs.). Geomorfologia: uma atualização de bases e conceitos. 5 ed. Rio de Janeiro: Bertrand Brasil, 2003. p. 93-148.

EASTMAN, J. R. IDRISI taiga Tutorial. Worcester: Graduate School of Geography, Clark University, 2009. 333p.

. IDRISI 32 release 2, guide to GIS and image processing. v. 2. Worcester: Clark Labs - Clark University, 2001. 161p.

Idrisi for Windows Versão 2: introdução e exercícios tutoriais. Porto Alegre: UFRGS/ Centro de Recursos Idrisi, 1998. 240p.

EMBRAPA. Centro Nacional de Pesquisa em Solos. Sistema Brasileiro de Classificação de Solos (SiBCS), 2013, 353p.

FERREIRA, J. C.; ROCHA, J.; TENEDÓRIO, J. A.; SOUSA, P. M. Ensaio de delimitação de corredores verdes na Área Metropolitana de Lisboa: integração de dados fuzzy através da análise multi-critério. In: ENCONTRO DE UTILIZADORES DE INFORMAÇÃO GEOGRÁFICA, 8., Oeiras, 2004. Anais ... Oeiras, Portugal: ESIG, 2004. p. 0-16.

FITZ, P. R. Geoprocessamento sem complicação. São Paulo: Oficina de Textos, 2008.

FLORENZANO, T. G. Cartografia. In: FLORENZANO, T. G. (Org.). Geomorfologia: conceitos e tecnologias atuais. São Paulo: Oficina de Textos, 2008. v. 4, p. 105-128.

JENKS, G. F. Optimal data classification for choropleth maps. Department of Geographiy, University of Kansas Occasional Paper, 1977.

KERRY, R.; OLIVER, M. A. Comparing sampling needs for variograms of soil properties cmputed by the method of moments and residual maximum likelihood. Geoderma, v. 140, n. 4, p. 383-396, 2007.

MALCZEWSKI, J.. GIS $\square$ based land-use suitability analysis: a critical overview. Progress in Planning, v. 62, n. 1, p. 3-65, 2004.

MCCUEN, R. H. Hydrologic analysis and design. New Jersey: Prentice-hall, 1998. $814 \mathrm{p}$.

MIRANDA, J. I. Modelo de dados. In: MIRANDA, J. I. Fundamentos de Sistemas de Informações Geográficas. 2. ed. Brasília, DF: Embrapa Informação Tecnológica, 2010. p.115-1138.

MOREIRA, F. R.; BARBOSA, C.; CÂMARA, G.; ALMEIDA FILHO, R. Inferência geográfica e suporte à decisão. In: CÂMARA, G.; DAVIS JUNIOR, C. A.; MONTEIRO, A. M. V. Introdução à ciência da geoinformação. São José dos Campos: INPE, 2001. p.1-49.

SAATY, T. L. How to make a decision: the analytic hierarchy process. European Journal of Operational Research, v. 48, n. 1, p. 9-26, 1990.

SAATY, R. W. The analytic hierarchy process: what it is and how it is used. Mathematical Modelling, v. 9, n. 3-5, p. 161-176, 1987.

SAATY, T. L. A scaling method for priorities in hierarchical structures. Journal of Mathematical Psychology, v. 15, n. 3, p. 234-281, 1977. 
SARTORI, A. Avaliação da classificação hidrológica do solo para a determinação do excesso de chuva do método do Serviço de Conservação do Solo dos Estados Unidos. 2004. Dissertação (Mestrado em Engenharia Civil) - Faculdade de Engenharia Civil, Arquitetura e Urbanismo, Universidade Estadual de Campinas, Campinas, 2004.

SARTORI, A.; LOMBARDI NETO, F.; GENOVEZ, A. M. Classificação hidrológica de solos brasileiros para a estimativa da chuva excedente com o método do Serviço de Conservação do Solo dos Estados Unidos. Parte 1: Classificação. Revista Brasileira de Recursos Hídricos, v. 10, n. 4, p. 05-18, 2005.

SCS - Soil Conservation Service. Hydrology. Section 4 - Hydrology, Chapter 10 - Estimation of direct runoff from storm rainfall. In: National Engineering Handbook. Washington: USDA, 1972. p.101- 1023.

SOUZA, M. J. N.; OLIVEIRA, V. P. V. Os enclaves úmidos e subúmidos do semiárido do Nordeste brasileiro. Mercator, Fortaleza. v. 5, n. 9, p. 85-102, dez. 2006.

ZADEH, L. A. Fuzzy sets. Information and Control, v. 8, p. 338-353, 1965.

Augusto César Praciano Sampaio - Geógrafo, mestre em Geografia Física e especialista em Geoprocessamento pela Universidade Estadual do Ceará. Atualmente é pesquisador no Instituto Nacional de Pesquisas Espaciais, atuando na área de modelagem espacial. https://orcid.org/0000-0003-2309-4292

Frederico de Holanda Bastos - Doutor em Geografia e mestre em Desenvolvimento e Meio Ambiente pela Universidade Federal do Ceará e especialista em Geoprocessamento e Bacharel/Licenciado em Geografia pela Universidade Estadual do Ceará. Atua na área de Geomorfologia e Geografia Ambiental. Professor Adjunto e Docente Permanente do Programa de Pós-graduação em Geografia da Universidade Estadual do Ceará. https://orcid.org/0000-0002-4330-7198

Abner Monteiro Nunes Cordeiro - Geógrafo, doutor em Geografia Física pela Universidade Estadual do Ceará. Atua na área de planejamento ambiental com ênfase em estudos de planos diretores e na área de Geografia Física com destaque nos estudos ambientais integrados e geomorfológicos. https://orcid. org/0000-0002-4867-7083

\section{Contribuição dos autores}

Todos os autores ofereceram substanciais contribuições científicas e intelectuais para o desenvolvimento do estudo. As tarefas de concepção, preparação e redação do manuscrito, bem como revisão crítica, foram desenvolvidas em grupo. O autor Augusto César Praciano Sampaio foi o idealizador do artigo, sendo o responsável pelo desenvolvimento teórico, 
conceitual e científico. O segundo e o terceiro autores fizeram acréscimos e correções fundamentais a partir de suas experiências em pesquisa científicas, além da estruturação e da revisão textual. Portanto, o texto final expressa um trabalho coletivo realizado ao longo da pesquisa de mestrado do primeiro autor, defendida em fevereiro de 2018, no Programa de Pós-Graduação em Geografia da Universidade Estadual do Ceará, sob orientação do professor Frederico de Holanda Bastos e co-orientação do professor Abner Monteiro Nunes Cordeiro.

Recebido para publicação em 15 de agosto de 2018 Aceito para publicação em 5 de outubro de 2018 